\title{
De qué conversan los periodistas españoles en Twitter. Contenidos y tendencias en la twitteresfera española
} What are the Spanish journalists talking about on Twitter.
Content and trends in the Spanish twittersphere

\author{
Gabriel Jaraba; Santiago Tejedor
}

Cómo citar este artículo:

Jaraba, Gabriel; Tejedor, Santiago (2020). “De qué conversan los periodistas españoles en Twitter. Contenidos y tendencias en la twitteresfera española". El profesional de la información, v. 29, n. 1, e290118.

https://doi.org/10.3145/epi.2020.ene.18

Artículo recibido el 09-06-2019 Aceptación definitiva: 18-11-2019

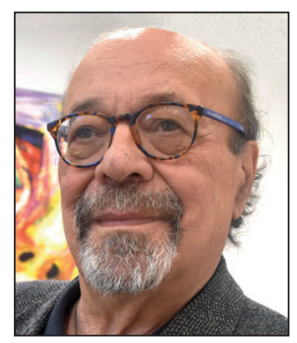

Gabriel Jaraba

https://orcid.org/0000-0002-6392-1566

Universitat Autònoma de Barcelona Departamento de Periodismo y Ciencias de la Comunicación

Campus UAB, Bellaterra.

08193 Cerdanyola del Vallés

(Barcelona), España

gabriel.jaraba@uab.cat

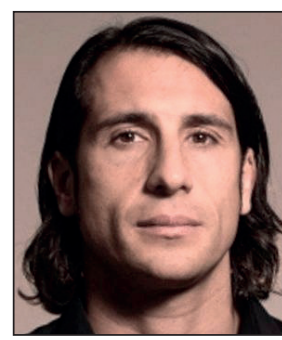

Santiago Tejedor $\square$

https://orcid.org/0000-0002-5539-9800

Universitat Autònoma de Barcelona

Departamento de Periodismo y Ciencias de

la Comunicación

Campus UAB, Bellaterra.

08193 Cerdanyola del Vallés

(Barcelona), España

santiago.tejedor@uab.cat

\section{Resumen}

El consumo de noticias se ha convertido en una experiencia social. En este escenario nos planteamos responder al interrogante: ¿De qué conversan los periodistas españoles en Twitter? Este trabajo estudia qué tipo de contenido emiten en Twitter para establecer si la vinculación de estos profesionales con los medios convencionales o con los cibermedios influye en la orientación temática de su actividad en Twitter. Se ha trabajado con tres muestras (portadas de cinco diarios de referencia de España, diez cuentas de Twitter de periodistas de medios de comunicación tradicionales y diez cuentas de ciberperiodistas). La investigación, de dos años de duración, ha analizado 3.500 tuits a partir de un sistema de clasificación de unidades de registro conformado por 35 categorías temáticas. El estudio concluye, entre otros aspectos, que la política nacional e internacional es el gran tema de Twitter junto a una preocupación creciente por el terrorismo yihadista y los asuntos del contexto iberoamericano por encima del europeo. El trabajo advierte también de la existencia de ciertas brechas temáticas sobre sociedad, cultura, arte o ciencia. Además se detecta una tendencia creciente del uso de la plataforma con fines de autopromoción por parte de los periodistas.

\section{Palabras clave}

Twitter; Periodismo; Periodistas; Prensa; Diarios; Medios convencionales; Legacy media; Cibermedios; Medios sociales; Agenda; Información; Internet; Poder; Comunicación; España.

\footnotetext{
Abstract

The consumption of news has become a social experience. In this scenario, we set out to answer the following question: What are Spanish journalists talking about on Twitter? This work studies what type of content they emit on Twitter to establish if the link between these professionals and the mass media or with the cybermedia influences the thematic orientation of their tweet. The study has worked with three samples (a sample of covers of five newspapers of reference of Spain, a sample of ten accounts of Twitter corresponding to journalists from mass communication media and a sample of ten accounts of cyberjournalists). The two-year research has analyzed 3.500 tweets based on a classification system of registration units made up of 35 thematic categories. The study concludes, among other aspects, that politics (national and international) is the great theme of Twitter along with a growing concern for jihadist terrorism and issues of the Ibero-American context over Europe. The work also warns of the existence of thematic gaps in certain areas (such as society, culture, art or science). In addition, an increasing tendency of the use of the platform for the purpose of self-promotion by journalists is detected.
} 


\section{Keywords}

Twitter; Journalism; Journalists; Press; Newspapers; Legacy media; Cybermedia; Social media; Agenda; Information; Internet; Power; Communication; Spain.

\section{Introducción}

La sociedad siempre ha sido una red en una u otra medida. Lewin (1947) señaló que la percepción, el comportamiento de los individuos y la misma estructura grupal a la que pertenecen se inscriben en un espacio social configurado por el grupo y su entorno. Los trabajos de autores clásicos como Moreno (1954), Granovetter (1974) o King y Wertheimer (2009) resaltan la importancia del concepto de red en la historia de la humanidad, al tiempo que advierten que la percepción de un objeto se realiza dentro de una totalidad compleja y organizada. La sociedad no es homogénea, sino que se articula alrededor de un conjunto de puntos (nodos) que están conectados. El concepto de red ha estado desde sus orígenes vinculado con el poder. El surgimiento de las redes globales de comunicación (Thompson, 1998), por ejemplo, hicieron posible una estructuración en clave imperial de los mercados. Las redes otra vez daban forma al poder.

La denominada "sociedad red" relaciona la evolución económica y las transformaciones políticas, sociales y culturales en el marco de la teoría integral de la comunicación (Castells, 1997). La información y los intercambios alrededor de ésta se ubican en el centro de este espacio reticular donde la comunicación está directamente vinculada al poder (Castells, 2006). Piscitelli señalaba que

"en internet se produce la emergencia de la complejidad, la interacción de elementos simples desprovistos de toda inteligencia, acaban por generar un cerebro y una piel globales, inesperadas y superpoderosas" (Piscitelli, 2005, p. 22).

El ciberespacio ha renovado y multiplicado el protagonismo del concepto de red en nuestras sociedades, así como el modo de estudiarlas (Del-Fresno-García; Marqués-Martínez; Sánchez-Paunero, 2014). El impacto del ciberespacio en el cambio social ha sido objeto de reflexión teórica bajo conceptos como los de "masas inteligentes" (Rheingold, 2002) o "alquimia de las multitudes" (Pisani; Piotet, 2009). Las revueltas populares de los países árabes en el primer trimestre de 2011 pusieron de relieve las repercusiones políticas de los nuevos medios (especialmente Twitter), así como su vínculo con el poder y con el periodismo (Sandiumenge, 2012).

En este escenario, Twitter se ha convertido en una red social ampliamente utilizada por los periodistas en su desempeño profesional por su interés como fuente informativa, por su potencial como plataforma de publicación y por su versatilidad como espacio dialógico. Sin embargo, Twitter no es estrictamente periodismo. Esta plataforma comunicativa estructurada como una red asimétrica de microblogging no posee un uso predeterminado. Desde el campo político (Gutiérrez-Rubí, 2013; Caldevilla et al., 2019) al periodístico (Jaraba, 2013; 2014; 2015), muchos trabajos han indagado sobre las posibilidades comunicativas de esta plataforma y su alcance en un escenario hiperconectado (Reig, 2011) que confiere un gran protagonismo a los

La cultura es un escenario en el que se percibe con mayor claridad la existencia de incipientes tendencias renovadoras, aperturistas o de futuro en Twitter

usuarios (Gillmor, 2004; Nafría, 2007) y que viene marcado por la desintermediación (Orihuela, 2015). Reig, Mancinas-Chávez y Nogales-Bocio (2017) proponen, a partir de la metodología compleja de Edgar Morin o de lo que Konrad Lorenz llamó epistemología comparada, una "Metodología del enfoque estructural complejo" (MENEC) o "Enfoque estructural complejo" (EEC) donde todo está relacionado. Este conjunto de propuestas, procedentes de diferentes áreas y enfoques, resulta de gran validez para el estudio de nuevos fenómenos comunicativos como son las redes sociales.

Twitter reúne una serie de características muy particulares a nivel comunicativo. El componente de inmediatez (Cambronero, 2012), la sencillez, la movilidad en la producción y la asimetría de relaciones entre sus usuarios (Orihuela, 2011) la convierten en un "espacio" comunicativo innovador y con grandes potencialidades en el actual escenario comunicativo que cuestiona la teoría de la agenda-setting de McCombs (McCombs; Masel-Walters, 1976; McCombs, 1994; McCombs; Evatt, 1995). Este artículo responde a la siguiente pregunta de investigación de ámbito descriptivo: “¿De qué conversan los periodistas españoles en Twitter?". Con relación a ella, en el marco del trabajo, la investigación persigue los siguientes objetivos:

- Analizar qué tipo de contenido emiten los periodistas españoles en Twitter y comprobar si varía respecto a si se trata de periodistas que trabajan en medios convencionales o si lo hacen en cibermedios.

- Establecer una posible diferencia de tendencias en el contenido a partir de la adscripción profesional a un medio convencional o un cibermedio.

- Definir en qué consisten estas tendencias y de qué modo se expresan en el contexto profesional e informativo español.

\section{Marco teórico}

El consumo de noticias se ha convertido en una experiencia social (Tejedor; Carniel-Bugs; Giraldo-Luque, 2018). Los contenidos informativos se han transformado en piezas "portátiles", "personalizadas" y "participativas" (Matsa; Mitchell, 2014). En este contexto, se observa una preocupación por analizar el impacto del ciberespacio en la dieta informativa de 
los usuarios. Desde los trabajos de Levy (1999; 2007) sobre la consolidación de una nueva cultura digital, las reflexiones de Martín-Serrano (2008) sobre las mediaciones sociales, o las investigaciones de Galindo-Cáceres (2010a; 2010b; 2011) respecto al comportamiento -previsible y pertinente- de los usuarios en las redes sociales, se ha venido cuestionando la teoría de la agenda-setting teorizada por McCombs (McCombs, 1992; McCombs; Shaw, 1972; 1976) y su impacto en la ciudadanía digital. Autores como Manovich (2005) afirman que existe una dependencia de los nuevos medios respecto de las convenciones fijadas por los viejos. En esta misma línea, Martín-Barbero (2015) señala que el paradigma digital se vincula directamente con modelos anteriores. A ello se une la emergente necesidad de una alfabetización digital y mediática (Singh et al., 2015) que permitan afrontar los nuevos desafíos formativos que demanda el ciberespacio en un escenario marcado por la esencia líquida de los procesos económicos, políticos, culturales y educativos que se encuentran estrechamente ligados con las dinámicas informativas y comunicativas (Bauman, 2003).

Las redes sociales, identificadas como servicios basados en aplicaciones web que permiten al usuario interactuar (Gentile et al., 2012) a través de un perfil propio (Boyd; Ellison, 2008; Lenhart et al., 2007), se han convertido en plataformas de gran importancia en el ecosistema mediático actual (Bulut; Doğan, 2017). Autores como Boyd (2007), O'Keeffe y Clarke-Pearson (2011) o Akter y Nweke (2016) han destacado que la participación del usuario en los medios sociales ofrece la posibilidad de ampliar su visión del mundo, de su comunidad y de sí mismos.

Piscitelli, Adaime y Binder (2010), Colás-Bravo; González-Ramírez y De-Pablos-Pons (2013), Bernal-Bravo y Angulo-Rasco (2013) y Pérez-Tornero et al. (2015), entre otros, han incidido en el protagonismo que estas redes poseen entre los jóvenes, mientras que trabajos como el de Schwarz (2011) apuntan al abandono de ciertos espacios de comunicación tradicional en beneficio de la comunicación instantánea que potencian las redes.

El componente de la sociabilidad (Quan-Hasse; Young, 2010) unido a la oportunidad de crear redes propias de contactos y con internautas que posean intereses afines es otro de los elementos definitorios de estas plataformas colaborativas (Shao, 2009; Sheldon, 2009; Papacharissi; Mendelson, 2011; Lin; Lu, 2011; Cheung; Chiu; Lee, 2011; Baek et al., 2011; Ruano; Congote; Torres, 2016). La posibilidad de compartir información entre internautas (Alhabash et al., 2012; Chung et al., 2016), la amplia amalgama de recursos informativos existentes, la capacidad de seleccionar recursos (Martínez-Serrano; Ferraz-da-Cunha, 2016) y el intercambio de éstos (Sharma; Verma, 2015) conforman las principales motivaciones para los usuarios, al tiempo que inciden directamente en su dieta informativa (Kurtulus; Ozkan; Öztürk, 2015). A ello se unen la versatilidad comunicativa de las plataformas (Pempek; Yermolayeva; Calvert, 2009), la invitación perenne a la integración, al intercambio (Sheldon, 2009; Bulut; Doğan, 2017), o al refuerzo del propio branding o marca personal (Molyneux, 2014, Hanusch; Bruns, 2017) como periodista presente en las redes sociales.

Las reflexiones de Reig en La telaraña mediática (2010) y Los dueños del periodismo (2011) aluden a la existencia de un entramado de poderes socioeconómicos que alcanzan a los medios de comunicación y, al mismo tiempo, advierten de la existencia de medios y grupos de comunicación que invierten en negocios ajenos a su teórica razón social. Partiendo de este enfoque, el estudio se propone analizar de qué tipo de temas y desde qué enfoque publican los periodistas españoles en Twitter para intentar desgajar posibles tendencias en los contenidos distribuidos a través de esta red social. López-Meri (2015) apunta que las noticias serían, en determinados contextos informativos, fruto de la negociación pública y en tiempo real entre periodistas y usuarios que ejercen de fuentes.

\section{Metodología}

La investigación, de tipo descriptivo y analítico, posee un enfoque cuantitativo y cualitativo. El estudio ha analizado el contenido de 3.500 tweets entre 2017 y 2018 a partir del análisis del contexto de los propios flujos de tweets y del estado de la actualidad informativa de acuerdo con lo que expresan las portadas de periódicos de referencia. Siguiendo la clasificación de la investigación social de Berganza-Conde y Ruiz-San-Román (1992), el trabajo presenta una investigación longitudinal retrospectiva que estudia una sucesión diacrónica de mensajes vía Twitter producidos a lo largo de unas franjas de tiempo y analiza las portadas de periódicos publicadas en los mismos períodos. Las fuentes de las que proceden los datos obtenidos del seguimiento de los dos grupos de periodistas se estructuran en dos muestras invitadas y no probabilísticas. La metodología empleada es la propia del análisis de contenido a partir de los aportes de autores clásicos como Berelson (1971), Krippendorff (1990) y Holsti (1969), y aplicado al ciberespacio según las propuestas de Vilches (2011).

Para conseguir llevar a cabo la adaptación metodológica al escenario de internet, convertimos en operativos elementos cuantitativos. Para ello, se ha diseñado un sistema de categorización de unidades de registro que posteriormente han sido cuantificadas. La sistematización de ese proceso desemboca en una descripción de fenómenos y tendencias de base cuantitativa que, gracias a la simbolización realizada a través de la categorización y el análisis, accede a la dimensión cualitativa. Un elemento muy útil para el análisis cualitativo ha sido disponer del contexto proporcionado por la muestra de portadas de periódicos.

El núcleo de la investigación se centra en el análisis de contenido de dos muestras de cuentas de periodistas presentes en Twitter y de las portadas de diarios publicadas en el mismo período. El primer seguimiento constituye un trabajo de campo que recoge unidades de análisis en forma de tweets a partir de su emisión en los timelines de las cuentas de los periodistas observados. El segundo seguimiento procede de una muestra documental hemerográfica en la que cada 
portada es una unidad de análisis y cada espacio informativo de ella, una unidad de registro. El análisis de contenido se realiza a partir de una categorización común a todos materiales mencionados, expresada en criterios temáticos relativos a la actualidad, según la tradición periodística de clasificación del contenido informativo surgida con el periodismo moderno del siglo XX y que ha evolucionado hasta las divisiones temáticas que presentan hoy tanto medios tradicionales como cibermedios.

La determinación de las unidades de análisis y de registro se ha producido como sigue:

- Una unidad de análisis formada por la muestra de portadas de periódicos seleccionados, publicados durante el período de seguimiento.

- Dos unidades de análisis formadas por las muestras de cuentas de Twitter con los tweets de los periodistas de medios convencionales (legacy media) y los periodistas de diarios digitales seleccionados.

La determinación de las unidades de registro se ha realizado como sigue:

- Cada tweet de cada muestra es una unidad de registro, considerando como tal el contenido textual del tweet y los eventuales elementos gráficos que puede incorporar.

- En las portadas de periódicos se considera unidad de registro cada elemento informativo de las mismas. Se excluyen los elementos publicitarios y las llamadas a páginas del interior que se refieren a contenidos de suplementos o páginas especiales que no tienen relación con la actualidad del día.

- La codificación de las unidades de registro es homogénea para todas las unidades de análisis. Consiste en la asignación de categorías y subcategorías a cada una de ellas.

\subsection{Corpus de estudio y muestras}

El corpus de estudio está formado por 3.500 tweets emitidos por 20 periodistas titulares de cuentas de Twitter entre enero de 2017 y marzo de 2018 y por 35 portadas de periódicos publicadas esos mismos días.

Se han diseñado tres muestras:

- Las portadas de cinco diarios de referencia, publicadas en el periodo estudiado.

- Diez cuentas de Twitter correspondientes a periodistas en ejercicio de medios de comunicación de masas convencionales.

- Diez cuentas de Twitter correspondientes a periodistas que trabajan exclusivamente en cibermedios. A estos se les ha identificado como "ciberperiodistas" o "periodistas digitales", si bien hoy día todo el periodismo es digital, para diferenciarlos de aquellos que integran la plantilla de un medio generalista de larga trayectoria y con presencia fuera del ciberespacio. A pesar de su muerte en 2017, se decidió mantener por su relevancia, trayectoria y actividad en Twitter, al periodista Miguel-Ángel Bastenier.

La muestra de portadas de periódicos la componen El país, El mundo, La razón, La vanguardia y El periódico. Se trata de tres diarios publicados en Madrid y dos en Barcelona, cuyas líneas informativas y editoriales abarcan la centralidad de las temáticas informativas mayormente publicadas y seguidas en España. No se ha incorporado un periódico de referencia como $A B C$ porque su portada es monotemática y a menudo de tipo editorial, con lo que su valor informativo en cuanto a variedad y representatividad es menor. Tampoco se han incluido los dos diarios publicados únicamente en catalán en Barcelona, El Punt Avui y Ara porque su opción informativa suele estar restringida a Cataluña e incorporan poca información internacional, con lo que adolecen igualmente de variedad y representatividad.

Cada portada de periódico, en tanto que unidad de análisis, incluye diversas unidades de registro. Cada espacio noticioso de la misma (titular, fotonoticia, titular con lead, sumario informativo, cintillo) es una unidad de registro. Quedan excluidos los espacios de autopromoción del diario (llamadas a cuadernillos especializados o suplementos, avances de suplementos dominicales, etc.) y toda inserción no estrictamente informativa sobre la actualidad del día, así como la publicidad. Cada unidad de registro incluye una categoría y una o varias subcategorías. Las categorías se adjudican de acuerdo con criterios temáticos y las subcategorías se establecen con intención descriptiva del contexto del contenido y de sus protagonistas.

\subsection{Muestra de periodistas de medios convencionales y de periodistas digitales}

Las dos muestras de periodistas, los que trabajan en medios clásicos y los que lo hacen en cibermedios, son muestras invitadas no probabilísticas (tabla 1). Ha sido necesario por tanto justificar la elección de los periodistas que las forman. Se han empleado cuatro criterios para identificar la pertinencia de la inclusión:

- experiencia y trayectoria en el ejercicio de la profesión;

- adscripción a un medio influyente (tanto entre los medios convencionales como entre los cibermedios) y pluralidad de los medios representados en su conjunto;

- antecedentes denotativos de reflexión sobre el periodismo, los medios y la comunicación;

- representatividad en mayor o menor grado de: grupo profesional generacional, tendencia en la línea informativa y la opinión, ejercicio del periodismo generalista y no especializado.

Los periodistas que integraron la muestra se presentan en la tabla 1. 
Tabla 1. Muestra de periodistas seleccionados

\begin{tabular}{|c|c|}
\hline Periodistas de medios convencionales & Periodistas de cibermedios \\
\hline Miguel A. Bastenier (@MABastenier) & Ignacio Escolar (@iescolar) \\
\hline Andreu Farràs (@afarrasc) & Montserrat Domínguez (@MontseHuffPost) \\
\hline Jordi Évole (@jordievole) & Silvia Cobo (@silviacobo) \\
\hline Francisco Marhuenda (@pacomarhuenda) & Principia Marsupia (@pmarsupia) \\
\hline Ana Pastor (@_anapastor_) & Rosa María Artal (@rosamariaartal) \\
\hline Hermann Tertsch (@hermanntertsch) & Ricardo Galli (@gallir) \\
\hline David Jiménez (@DavidJimenezTW) & Ramón Lobo (@ramonlobo) \\
\hline Mayka Navarro (@maykanavarro) & Manuel M. Almeida (@mmeida) \\
\hline Carlos Cuesta (@carloscuestaEM) & Iñigo S. Ugarte (@Guerraeterna) \\
\hline Carlos E. Cué (@carlosecue) & Gumersindo Lafuente (@sindolafuente) \\
\hline
\end{tabular}

\subsection{Tabla de categorías}

La tabla de categorías viene estructurada en grupos, en aras de la operatividad (tabla 2). En total se han definido 6 grupos temáticos que engloban 35 categorías.

Tabla 2. Categorías temáticas para el análisis

\begin{tabular}{|c|c|c|}
\hline Política & Internacional & Sociedad \\
\hline $\begin{array}{l}\text { Política española } \\
\text { Gobierno } \\
\text { Corona } \\
\text { Instituciones } \\
\text { Corrupción } \\
\text { Terrorismo }\end{array}$ & $\begin{array}{l}\text { Política internacional } \\
\text { Crisis internacionales } \\
\text { Yihadismo } \\
\text { Derechos humanos }\end{array}$ & $\begin{array}{l}\text { Problemas sociales } \\
\text { Economía } \\
\text { Trabajo } \\
\text { Industria } \\
\text { Sanidad } \\
\text { Educación } \\
\text { Solidaridad } \\
\text { Ecología }\end{array}$ \\
\hline Comunicación & Cultura & Miscelánea \\
\hline $\begin{array}{l}\text { Medios } \\
\text { Medios en política } \\
\text { Periodismo } \\
\text { Internet }\end{array}$ & $\begin{array}{l}\text { Instituciones culturales } \\
\text { Artes } \\
\text { Religión } \\
\text { Historia } \\
\text { Ciencia }\end{array}$ & $\begin{array}{l}\text { Hechos diversos } \\
\text { Interés humano } \\
\text { Espectáculo } \\
\text { Humor } \\
\text { Delincuencia } \\
\text { Catástrofes } \\
\text { Información local }\end{array}$ \\
\hline
\end{tabular}

\section{Resultados}

El estudio ofrece una panorámica general de los contenidos analizados y al mismo tiempo describe una serie de tendencias temáticas generales a través de los grupos de categorías. La tabla 3, que presenta el número de tweets por los grupos de categorías definidos, advierte del predominio de la política sobre el resto de temáticas. Las referencias a cuestiones políticas, junto a las de internacional, alcanzan unas cifras que llegan a minimizar los demás centros de interés.

Tabla 3. Panorama general y valores totales

\begin{tabular}{|l|c|c|c|c|c|c|}
\cline { 2 - 7 } \multicolumn{1}{c|}{} & Política & Internacional & Sociedad & Comunicación & Cultura & Miscelánea \\
\hline $\begin{array}{l}\text { Periodistas de medios } \\
\text { convencionales }\end{array}$ & 759 & 723 & 44 & 476 & 70 & 34 \\
\hline $\begin{array}{l}\text { Ciberperiodistas } \\
\text { digitales }\end{array}$ & 792 & 120 & 64 & 238 & 53 & 106 \\
\hline
\end{tabular}

Por su parte, en el caso de los periodistas de medios convencionales (gráfico 1), destaca el alto interés prestado a los asuntos internacionales. Cuando se examina el contenido de los tweets, se comprueba que la mayoría de las veces el recurso a la política internacional responde a la intención de dirimir en este terreno cuestiones relativas a la política española, por alusiones o por trasposición de hipotéticas semejanzas. Por ejemplo, noticias o comentarios relacionados con Venezuela, que sirven de supuesto apoyo a argumentaciones que tienen como objetivo la crítica a determinados partidos o personas de nuestro país, un asunto que se viene dando tanto en los medios de comunicación de España como en los ambientes políticos españoles y que ha llegado a ser incluso un lugar común. La diferencia de proporciones en este asunto entre periodistas de medios convencionales y ciberperiodistas (según la dife-

La política internacional sirve para dirimir cuestiones relativas a la política española, por alusiones o por trasposición de hipotéticas semejanzas 
renciación realizada en este estudio) denota que estos últimos recurren mucho menos (unas seis veces menos) al mencionado estilo de argumentación y circunscriben la actualidad internacional en la esencia o naturaleza del ámbito.

Otra divergencia temática destacable se da en el grupo de categorías correspondiente a comunicación. Hay que señalar que los tweets relacionados con la comunicación, en el caso de los periodistas de medios clásicos, no están orientados a tratar con la situación de los medios o el periodismo en general. La mayoría de ellos responden a estrategias de autopromoción. En la muestra que conforma este estudio, los ciberperiodistas suministran más información o invitan a la reflexión, mientras que los periodistas de medios tienden a una utilización de Twitter como plataforma de promoción propia.

En el grupo de categorías correspondiente a sociedad, se observa también una mayor inclinación informativa por parte de los periodistas de cibermedios. Concretamente, se percibe una mayor atención a asuntos noticiables relacionados con realidades que se desmarcan de la polarización hacia la política y sus polémicas. Sin embargo, esta atención responde a centros de interés de variedad reducida. El interés por lo social no llega a compensar la polarización política y no abre espacio suficiente a la aparición de tendencias de apertura y futuro.

La comparación de todas las categorías de los tweets en su conjunto ofrece una visión temática más detallada que permite comparar estos elementos diacrónicamente, columna a columna y en días sucesivos. Se aprecia que los periodistas (tanto de medios como de cibermedios) expresan sus contenidos abarcando un mayor número de categorías que las portadas. De este modo, se desprende que los periodistas en Twitter se ocupan de cuestiones de mayor amplitud temática que los contenidos que conforman la corriente principal de actualidad de las portadas. En las portadas de los diarios seleccionados (tabla 4), la categoría de política española (con 51 menciones), la de yihadismo (con 18), y política internacional y deportes (con 16 cada una) son las más frecuentes. Por el contrario, con una sola mención las de derechos humanos, religión, crisis internacionales, problemas sociales, gobierno y terrorismo ocupan los últimos lugares del análisis.

La información local ocupa un papel ínfimo. Ninguno de los actores que integran la muestra trata cuestiones relativas a este tipo de contenido (tabla 5), a diferencia de lo que hacen algunos diarios, concretamente los que se publican en Barcelona. Resulta llamativa la ausencia de información local en un medio de microblogging que teóricamente facilita la aproximación a realidades cercanas e incluso microrrealidades tanto geográficas como temáticas. De hecho, el estudio constata que los periodistas de Twitter, tanto de medios convencionales como de cibermedios, tienen un concepto de actualidad que se corresponde con el de la llamada "prensa nacional", caracterizada por:

- primacía de la política, especialmente nacional;

- atención a la política en proporciones muy parecidas a las de su presencia en los medios de masas;

- distancia considerable entre la cantidad de contenidos dedicados a política y los correspondientes a temas sociales, y más todavía a los culturales, artísticos, científicos, de interés humano y de sociedad en general;

- escasa presencia de deporte, al contrario de lo que sucede en la totalidad de los medios;

- escasa presencia de temas económicos en contraposición a la prensa impresa, no sólo por lo que respecta a los diarios especializados sino a las propias secciones de información económica de la prensa diaria.

Tabla 5. Temas (en cifras) de las portadas y de las muestras de periodistas

\begin{tabular}{|c|c|c|c|c|c|c|}
\hline & \\
\hline & Política española & Yihadismo & Internacional & Deportes & Artes & Economía \\
\hline $\begin{array}{l}\text { Periodistas de medios } \\
\text { convencionales }\end{array}$ & 659 & 175 & 496 & 12 & 26 & 14 \\
\hline \multirow[t]{2}{*}{ Ciberperiodistas } & 770 & 53 & 34 & 5 & 36 & 23 \\
\hline & Local & Ecología & Ciencia & Sanidad & Interés humano & \\
\hline $\begin{array}{l}\text { Periodistas de medios } \\
\text { convencionales }\end{array}$ & 0 & 8 & 8 & 5 & 2 & \\
\hline Ciberperiodistas & 0 & 8 & 8 & 5 & 5 & \\
\hline
\end{tabular}


La polarización alrededor de la política, que es común tanto en los ciberperiodistas como en los periodistas de medios clásicos, denota una homogeneización. A ello se añade que ambos emplean en una amplia mayoría la información internacional a manera de parafraseo de cuestiones políticas nacionales, un intento de argumentación por temática interpuesta. Los tweets referidos al yihadismo aparecen en mayor cantidad en la muestra de ciberperiodistas. En este caso no existe el efecto de "argumentación por elevación" propio de los tweets de política internacional, sino que se da una actitud de protesta ante los atentados yihadistas ocurridos en Europa (que es más intensa que en los periodistas de medios tradicionales).

El contraste de las categorías que aparecen en la muestra de portadas de periódicos y las de las dos muestras de periodistas de Twitter, establece que los ciberperiodistas prestan menos atención a la actualidad deportiva que los periodistas de medios, pero más a las artes. La mayor atención de los ciberperiodistas a los asuntos económicos se debe igualmente al efecto protesta, pues son tweets muy críticos con asuntos de la actualidad económica. Lo mismo sucede con la ecología, pero no con la sanidad, que podría haber sido considerada de modo parecido. Las semejanzas de proporciones en categorías como ciencia o temas de interés humano pueden interpretarse como la existencia de actitudes parecidas entre ambas muestras por un distanciamiento de las zonas más propicias a la polémica o la polarización ideológica.

La visión de conjunto de las categorías englobadas en el grupo "Política" ("Política española", "Gobierno", "Corona", "Instituciones", "Corrupción" y "Terrorismo") acentúa la percepción de la hegemonía de la polémica política como tendencia dominante entre los periodistas españoles en Twitter (tabla 6). Son los tweets sobre el debate político cotidiano los que predominan sobre cuestiones como la actuación del Gobierno, las actividades de la Corona, el papel de las instituciones o incluso la corrupción y el terrorismo. Se puede por tanto afirmar que tanto los periodistas de medios como los ciberperiodistas no desatienden otros asuntos, pero tienden a una actividad en Twitter de urgencia mediante la cual tratan de estar presentes en la Red acentuando su faceta más polémica e incluso retadora (Imágenes 1 y 2 ).

Sorprende que solamente dos tweets de la muestra de ciberperiodistas hayan sido categorizados en "Corrupción", cuando este asunto ocupa las portadas de los periódicos con frecuencia. Ello se explica -en las dos muestras de periodistas- por la tendencia general del contenido político en Twitter. El tono general de la polémica política, su aguda personalización y el modo como se centra en partidos e ideologías no abre un marco argumental suficiente para que se pueda dar una reflexión que aborde las cuestiones relacionadas con la corrupción de manera más profunda. Puntualicemos en todo caso que abunda el tema de la corrupción en los contenidos estudiados -sobre todo como trasfondo general de las polémicas- pero en un marco de polémica política agudamente personalizada y contextualizada en el debate general entre partidos.

Esta argumentación parece ser igualmente válida por lo que respecta a las categorías “Gobierno" e "Instituciones” y por los mismos motivos expuestos. Llama la atención por ello que, al abordar la categoría "Corona", nos encontremos con seis tweets en cada una de las dos muestras de periodistas, dado que la Corona de España aparece no como un ente colectivo, como es el caso de las instituciones en general, sino fuertemente personalizada en las figuras de la realeza. Es necesario recurrir aquí no sólo al contenido sino al contexto, que indica que la monarquía española genera de por sí muy pocas noticias aparte de los discursos del rey Felipe o de algún viaje o visita. Por otro lado, sólo tres tweets de las dos muestras de periodistas han sido categorizados en "Terrorismo". La suspensión de actividades por parte de ETA y el apaciguamiento de sus organizaciones frente a la sociedad civil ha supuesto que el terrorismo de matriz nacional haya desaparecido de la escena mediática. El terrorismo internacional que actualmente centra la preocupación general viene categorizado en este estudio como "Yihadismo". Los periodistas observados aquí hacen lo mismo que todos los de su profesión en nuestro país: dejar de
La polarización alrededor de la política denota una homogeneización en el tipo de contenidos difundidos en Twitter 
referirse a una cuestión que, como indican los sondeos generales de opinión, ha dejado de merecer la preocupación principal de los españoles.

Tabla 6. Clasificación categoría "Política" en los periodistas analizados

\begin{tabular}{|l|c|c|c|c|c|c|}
\hline \multicolumn{1}{|c|}{ Política } & Política española & Gobierno & Corona & Institucional & Corrupción & Terrorismo \\
\hline $\begin{array}{l}\text { Periodistas de medios } \\
\text { convencionales }\end{array}$ & 659 & 0 & 0 & 8 & 0 & 1 \\
\hline \multicolumn{1}{|c|}{ Política } & Política española & Gobierno & Corona & Institucional & Corrupción & Terrorismo \\
\hline Ciberperiodistas & 770 & 3 & 5 & 11 & 2 & 2 \\
\hline
\end{tabular}

En las categorías clasificadas como "Sociedad" ("Problemas sociales", "Economía, "Trabajo", "Industria”, "Sanidad", "Educación", "Solidaridad" y "Ecología"), las dos muestras de periodistas parecen igualmente atentos a los problemas sociales en general (tabla 7). Lo mismo sucede con los temas económicos, laborales, industriales y sanitarios. No hemos hallado referencias a la educación en las muestras estudiadas. El interés por la ecología es casi equiparable en las dos muestras, aunque un poco superior en la de periodistas digitales.

El conjunto denota una tendencia en los periodistas digitales de mayor atención y acercamiento a las distintas realidades sociales que se encuentran fuera del marco político e institucional, y en cantidades algo alejadas del gran peso de la política observado en el análisis anterior. Puede sorprender el desequilibrio observado en la categoría "Solidaridad" a favor de los periodistas de medios. Ello no se debe a que presten mayor atención a estos asuntos sino a una cuestión relacionada con el uso que hacen de Twitter estos últimos periodistas. La totalidad de los tweets categorizados en "Solidaridad" corresponden a una acción promocional de un programa de radio dedicado a una campaña solidaria. El motivo del uso de Twitter en este sentido viene motivado por la voluntad de promocionar el propio medio antes que de tratar asuntos solidarios con los que no existe una vinculación profesional. Esta última constatación obliga a recurrir al análisis de contenido aplicado para atender tanto al propio contenido como al contexto. Ciertamente, la categoría "Solidaridad" en la muestra de periodistas de medios engloba contenidos referidos a este asunto, pero la mera categorización temática no es lo suficiente y necesariamente explícita al respecto. Debemos contextualizar el contenido no sólo en cuanto a lo explícito del mismo sino por lo que respecta a la intención de su publicación. La aplicación de métodos de las ciencias sociales a tejidos tan fluidos y a veces sutiles como las redes sociales va a obligar cada vez más a dar cuenta de lo que sucede en ellas teniendo en cuenta parámetros y variables que requieren la posibilidad de matizaciones más precisas.

Entre las tendencias observadas, más allá de lo mencionado en este apartado, destaca la escasa atención a las cuestiones laborales, nula en el caso de los periodistas de medios convencionales, al igual que los asuntos sanitarios, que en este caso se refieren a la sanidad pública, y los educativos. Podría pensarse que un medio como Twitter, que permite un acercamiento a realidades sociales que se encuentran en la base de la mayoría de la población, sería un cauce adecuado para vehicular la necesidad de que los profesionales de la información den cuenta de ellas al público. Pesa más, en cambio, la tendencia de caracterización profesional general respecto al panorama mediático que determinadas intenciones que a veces se manifiestan. En todo caso, la tendencia de mayor acercamiento a lo social por parte de los periodistas digitales es un dato de interés.

Tabla 7. Clasificación categoría "Sociedad"

\begin{tabular}{|c|c|c|c|c|c|c|c|c|}
\hline \multicolumn{1}{|c|}{ Sociedad } & $\begin{array}{c}\text { Problemas } \\
\text { sociales }\end{array}$ & Economía & Trabajo & Industria & Sanidad & Educación & Solidaridad & Ecología \\
\hline $\begin{array}{l}\text { Periodistas de medios } \\
\text { convencionales }\end{array}$ & 6 & 14 & 0 & 1 & 0 & 1 & 14 & 8 \\
\hline Sociedad & $\begin{array}{c}\text { Problemas } \\
\text { sociales }\end{array}$ & Economía & Trabajo & Industria & Sanidad & Educación & Solidaridad & Ecología \\
\hline Ciberperiodistas & 17 & 23 & 2 & 5 & 5 & 0 & 3 & 8 \\
\hline
\end{tabular}

Las categorías de "Internacional" ("Política internacional", "Crisis internacionales", "Yihadismo", "Derechos humanos") denotan el gran peso de la política internacional en la muestra de periodistas de medios (tabla 8). El contraste con la misma categoría en la muestra de ciberperiodistas es sensible, tal y como sucede con la categoría "Yihadismo". Se observa un uso de la política internacional a manera de parafraseo de cuestiones políticas nacionales que denota un intento de argumentación por temática interpuesta. Esta tendencia se aprecia en menor medida en los ciberperiodistas. Las diferencias cuantitativas entre una y otra muestra en las categorías de este grupo indican un desmarque de los ciberperiodistas de esa actitud generalizada entre los periodistas de medios. La gran diferencia cuantitativa entre los datos que informan estas tendencias y los que corresponden a la categoría "Derechos humanos" advierte de la ausencia de atención a asuntos relacionados con el respeto y vulneración de los derechos humanos en el mundo reflejada en los tweets de ambas muestras de periodistas, que se centra en el seguimiento de esta cuestión básicamente en el panorama general de la actualidad internacional. La categorización de solamente cuatro tweets en "Derechos humanos" refuerza esta tendencia generalizada. 
Respecto a la categoría "Crisis internacionales", las que ocupan la atención de los periodistas son las que se dan entre las superpotencias y muy especialmente las de las zonas de Cercano Oriente inmersas en procesos bélicos. Sin embargo, hay una escasa atención hacia las cuestiones sobre Israel y Palestina y el mundo árabe en general. Zonas críticas como Pakistán-India, India-China con el añadido de Tíbet y la situación en Birmania -entre la junta militar y el gobierno de Aung San Suu Kyi- están ausentes del foco de atención. Ni siquiera la cuestión del Sahara, o Ceuta, Melilla y Gibraltar, merecen ni una sola mención. África está ausente del foco de atención, igual que Asia salvo, en parte, China, así como las cuestiones europeas generales y asuntos civilizacionales relacionados con la globalización. La política estadounidense, salvo sus zonas de roce crítico con Rusia, tampoco es objeto de atención ni en términos informativos ni de opinión, de modo que el seguimiento de los periodistas de medios y los periodistas digitales no aporta información ni elementos propios de una realidad tan determinante de la evolución global como es la estadounidense en términos políticos, económicos, culturales e institucionales, por no hablar de sus movimientos sociales, conflictos raciales y de inmigración, y de los asuntos relacionados con la concepción de la democracia, sus instituciones y la república.

Tabla 8. Clasificación categoría "Internacional”

\begin{tabular}{|l|c|c|c|}
\hline \multicolumn{1}{|c|}{ Internacional } & Política internacional & Crisis internacionales & Yihadismo \\
\hline $\begin{array}{l}\text { Periodistas de medios } \\
\text { convencionales }\end{array}$ & 496 & 65 & 175 \\
\hline \multicolumn{1}{|c|}{ Internacional } & Política internacional & Crisis internacionales & Yihadismo \\
\hline Ciberperiodistas & 34 & 32 & 53 \\
\hline
\end{tabular}

Los asuntos relacionados con "Comunicación" (clasificados en los epígrafes de "Medios", "Medios en política”, "Periodismo" e "Internet") ocupan una parte importante de la actividad en Twitter de las dos muestras de periodistas, siendo en cifras absolutas superior al de otros grupos de categorías (tabla 9). Es necesario, sin embargo, hacer de nuevo ciertas precisiones respecto al contenido y sus denotaciones reales. Los periodistas de medios tweetean más sobre estos que los ciberperiodistas (concretamente, cuatro veces más). No obstante, este alto nivel de tweets se debe a un aspecto señalado anteriormente: gran parte del uso de Twitter por parte de los periodistas está orientado a la propia promoción (imagen 3).

No encontramos en el contenido de los tweets de la categoría "Medios" un debate o conversación sobre cuestiones relativas al presente y futuro de los medios de comunicación. Se produce la misma paradoja que en otros espacios sociales, la de la ausencia de los profesionales del gran debate sobre las profundas transformaciones comunica-

Jordi Évole
Próximo domingo
VuelveSalvados
Cumplimos 10 años
Y por 1a vez
No te decimos con qué empezamos
Es mejor así
\#1DeCada5
(s) Salvados

Imagen 3. Uso de Twitter para promocionar programas propios cionales no sólo en el seno de las industrias del ramo sino en el panorama social general. Sí que se da algo parecido sin embargo en la categoría de "Periodismo", en la que aparece contenido que en una medida u otra responde a asuntos deontológicos o de formación en la profesión. Este aspecto se aprecia en mayor medida en la muestra de periodistas de medios -casi tres veces más que en la de ciberperiodistas - y dado que en esta categoría no nos encontramos ante usos instrumentales de Twitter en términos de promoción propia o de otros, podemos decir que la atención a cuestiones profesionales no viene mediatizada.

Sin embargo, estas cuestiones periodísticas que se tratan nunca aparecen relacionadas, ni entre los periodistas de medios ni en los digitales, con los asuntos que ocupan la preocupación de la profesión periodística en los grandes medios internacionales y que a veces se hacen públicas en forma de libro. En muchos tweets de una y otra muestra, se da a conocer la aparición de libros periodísticos, algunos muy notables, pero nunca se recomienda libro alguno que no sea en español, ni tampoco medio en cualquier otra lengua que incorpore reflexiones como las que acabamos de mencionar. A pesar de que muchos periodistas de las dos muestras leen inglés y algunos lo usan como lengua profesional, su actividad en Twitter es estrictamente hispánica en términos de centros de atención. La lengua y culturas francesa, digamos de paso, desaparecen del panorama que tratamos del mismo modo que lo han hecho de tantos otros lugares del espectro social.

La preocupación sobre cuestiones relacionadas con internet se presenta en cambio en un sentido inverso al de la categoría "Periodismo" por lo que respecta a las proporciones entre periodistas de medios y ciberperiodistas. Son estos los que tweetean más asuntos relacionados con la Red, casi tres veces más que los primeros. Parece lógico a primera vista que los periodistas digitales se ocupen más de cosas de internet, pero cabe recordar que los periodistas de medios que estudiamos figuran en esta investigación precisamente por el hecho de ser avezados usuarios de Twitter. 
La categoría "Medios en política", que abarca los tweets que recogen la actividad de los medios de comunicación directamente vinculada a la política es muy peculiar. Las cuestiones relativas a los medios entran en esta categoría cuando denotan una vinculación directa a la actualidad política, especialmente la electoral. Este aspecto justifica el motivo de crear esta categoría particular y no asociar los tweets a ella vinculados a las de "Política española" o a "Medios" en el marco de esta investigación.

Tabla 9. Clasificación categoría "Comunicación”

\begin{tabular}{|c|c|c|c|}
\hline \multicolumn{1}{|c|}{ Comunicación } & Medios & Medios en política & Periodismo \\
\hline $\begin{array}{l}\text { Periodistas de medios } \\
\text { convencionales }\end{array}$ & 218 & 156 & 21 \\
\hline Comunicación & Medios & Medios en política & Periodismo \\
\hline Ciberperiodistas & 66 & 73 & 26 \\
\hline
\end{tabular}

Existe por otro lado un descenso cuantitativo de los tweets relacionados con "Cultura" (bajo los ámbitos de "Cultura", "Artes", "Religión”, “Historia”, “Ciencias" y “Deportes”) respecto a las categorías precedentes (tabla 10). Persiste también en esta categoría la tendencia general que se revela como fundamental entre los periodistas estudiados: su asimilación al modelo general de comportamiento profesional que impera en los medios de comunicación de masas, de modo que la existencia de internet apenas altera las características de esa caracterización generalizada.

En las categorías de este grupo destaca cuantitativamente "Artes". Al incluir asuntos como actuaciones de artistas interpretativos, cine y teatro, entre otros, ocupa la mayor parte de las referencias por cuanto los tweets relacionados responden a la actualidad en este campo. Al mismo tiempo se observa una diferencia sensible respecto a los temas científicos, tratados cuantitativamente casi igual en ambas muestras, asuntos que no suelen ocupar excesivo espacio en los medios convencionales, cosa que confirma la tendencia indicada en el párrafo precedente.

Es interesante observar lo que sucede con la categoría "Religión". Las actividades del Vaticano y el papado católico no merecen la menor atención. Vuelve a darse en esta categoría otra característica del estado general de los medios: la ausencia de una información religiosa seria y variada, como sucede en tantos otros países. Nótese que habiendo cobrado la religión una especial importancia en los últimos años del siglo XX y primeros del XXI -y no sólo a causa del fundamentalismo islámico - esta ausencia de referencias de información religiosa se da no solamente relacionada con la Iglesia Católica. No encontramos mención alguna a otras religiones en un país en el que los inmigrantes latinoamericanos suelen nutrir nuevas congregaciones pentecostalistas. A ello habría que añadir las referencias reducidas al pueblo gitano, a los sikhs, que abundan en las grandes capitales, o a la amplia población musulmana no solamente inmigrante.

En la categoría "Deportes" hay que destacar que es mucho menor la actividad de tweets sobre deportes que sobre artes, puesto que los periodistas de las dos muestras son generalistas mientras que los profesionales que tratan de deportes en los medios están especializados en este campo. Así, en la muestra de periodistas de medios, las referencias deportivas están entre las cantidades más reducidas; mientras que los tweets de los periodistas digitales son más de la mitad que los de los anteriores. Con cifras tan reducidas es aventurado hacer inferencias al respecto, simplemente observar que aquí se da un indicio de mayor apertura temática por parte de los periodistas digitales (imagen 4).

\section{(6) Ana Pastor \\ @_anapastor_}

Siguiendo

Ojalá veamos a @MarcGasol en el All Star

2017 . Cada RT es un voto.

\#NBAVOTE

16:53 - 5 ene. 2017

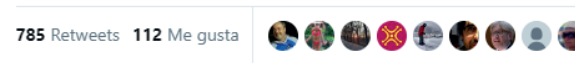

$\bigcirc \begin{array}{lllll}785 & \bigcirc & 112 \quad \square\end{array}$

Imagen 4. Ejemplo de tweet sobre deportes

Tabla 10. Clasificación categoría "Cultura"

\begin{tabular}{|c|c|c|c|c|c|c|}
\hline \multicolumn{1}{|c|}{ Cultura } & $\begin{array}{c}\text { Instituciones } \\
\text { culturales }\end{array}$ & Artes & Religión & Historia & Ciencia & Deportes \\
\hline $\begin{array}{l}\text { Periodistas de medios } \\
\text { convencionales }\end{array}$ & 4 & 26 & 8 & 5 & 8 & 12 \\
\hline Cultura & $\begin{array}{c}\text { Instituciones } \\
\text { culturales }\end{array}$ & Artes & Religión & Historia & Ciencia & Deportes \\
\hline Ciberperiodistas & 1 & 34 & 0 & 2 & 9 & 5 \\
\hline
\end{tabular}

La categoría de “Miscelánea” (que abarca: “Hechos diversos”, Interés humano”, “Espectáculo”, “Humor”, “Delincuencia”, "Catástrofes") recoge también elementos que aportan diversidad temática a los tweets (tabla 11). Al incluir asuntos considerados ligeros en el despliegue temático propio de los medios informativos -generalmente incluidos en la segunda mitad de la paginación de los periódicos- puede indicar interés por asuntos que en los medios convencionales serían considerados de poca importancia pero que en una red social adquieren un cariz distinto. En los últimos tiempos se viene observando en las ediciones digitales de los periódicos impresos -y muy especialmente en los medios informati- 
vos creados específicamente como cibermedios y posicionados para abrirse espacio entre los nuevos públicos que demandan contenidos informativos en la Red- la apertura a áreas temáticas que incluyen un mayor número de asuntos "ligeros" con el ánimo de incidir en los espacios de internautas que se mueven en las redes sociales. La aparición de la edición española del Huffington post o la evolución de las nuevas secciones de las ediciones digitales de El país marcan significativamente esta tendencia. Ello hace que materiales informativos que solían aparecer relegados o incluso descartados en los medios convencionales ocupen en los cibermedios un mayor espacio y apelen a una mayor atención.

Esta situación haría suponer que los periodistas que operan en Twitter podrían hallar en este nuevo interés, o por lo menos oportunidad ofrecida por las nuevas tendencias de los cibermedios, una vía para aproximarse a intereses más cercanos a los públicos más básicos de la Red mediante tweets sobre cuestiones que los medios convencionales no ayudan a difundir. Sin embargo, como hemos indicado en análisis previos, prevalece tanto en los periodistas de medios convencionales como en los ciberperiodistas, una inclinación temática más propia de los medios de comunicación de masas que de los cibermedios. Esto sucede igualmente entre los ciberperiodistas. La polarización política, institucional y partidaria concentra todos sus esfuerzos. Hay que decir que muchos de los medios digitales en los que colaboran parte de los profesionales que forman parte de la muestra de ciberperiodistas se publican con una clara intención de incidir en el campo de la información política, incluso de actuar como elementos altamente críticos en el medio de la política partidaria, con lo que los profesionales de nuestra muestra actúan en Twitter de modo idéntico a como lo hacen en sus medios.

La categoría "Catástrofes" también se ve influida por estas circunstancias y asimismo la mencionada consideración de Twitter como plataforma autopromocional por parte de algunos periodistas de medios. Esto se refleja en la mayor cantidad de tweets emitidos por una periodista de televisión cuyo medio estaba realizando una cobertura continuada de unos incendios producidos en Cantabria. En el caso de la categoría "Espectáculo", al no estar incluidos en ella los asuntos referentes a artes interpretativas, se recogen temas relativos a personajes famosos, concursos, etc. De este modo, los tweets incluidos en esta categoría pertenecientes a la muestra de periodistas de medios y su ausencia en la muestra de ciberperiodistas denota una diferencia de interés al respecto por parte de unos y otros. Las cifras parecidas de tweets referentes a "Interés humano" y "Hechos diversos", prácticamente equiparable en ambas muestras, muestra esa homogeneidad en la consideración de los temas ligeros que hemos indicado al inicio de este análisis en una y otra muestra de tweets.

Tabla 11. Clasificación categoría “Miscelánea”

\begin{tabular}{|c|c|c|c|c|c|c|}
\hline Miscelánea & Hechos diversos & Interés humano & Espectáculo & Humor & Delincuencia & Catástrofes \\
\hline $\begin{array}{l}\text { Periodistas de medios } \\
\text { convencionales }\end{array}$ & 8 & 2 & 5 & 0 & 5 & 32 \\
\hline Miscelánea & Hechos diversos & Interés humano & Espectáculo & Humor & Delincuencia & Catástrofes \\
\hline Ciberperiodistas & 11 & 5 & 0 & 6 & 0 & 15 \\
\hline
\end{tabular}

\section{Conclusiones}

Este estudio permite extraer una serie de conclusiones respecto al interrogante propuesto: ¿De qué hablan los periodistas españoles en Twitter?:

- La política es el gran tema: tanto los periodistas de medios convencionales como los de medios digitales centran prioritariamente su atención en la política nacional e internacional. Concretamente los segundos lo hacen en mayor medida que los primeros. Los contenidos de información y opinión políticas siguen un discurso y unos centros de interés idénticos a los que se usan en el conjunto del panorama mediático español. El contenido de los tweets sobre política española está sobre todo orientado a la opinión. Este aspecto conecta en cierto modo con los trabajos de LópezMeri (2015), que analizó el impacto y alcance de Twitter en el escenario informativo y, en esta línea, la existencia de un intercambio o negociación entre los profesionales de la información y los propios internautas. Por su parte, los periodistas de medios convencionales publican tweets sobre asuntos internacionales en gran medida para "tirar por elevación" hacia contenidos de política española. Tanto éstos como los periodistas digitales que forman las muestras siguen en su mayoría un modelo de actitud profesional perfectamente asimilable al tipo de medios de comunicación de masas hegemónicos en España y al del periodismo que en ellos se practica. Esto sería comprensible con la muestra de periodistas de medios convencionales, pero también se da en la muestra de periodistas digitales.

Existen sin embargo algunas brechas temáticas y de orientación informativa que señalan tendencias de desmarque del mencionado modelo hegemónico, pero el altísimo volumen de interés por los temas políticos, que genera a nivel institucional Madrid, les aproxima a la citada tendencia mayoritaria. El uso prioritariamente instrumental que los periodistas hacen de Twitter, muy especialmente los de medios convencionales, hace que sus tweets se ocupen en gran parte de vehicular información y debate políticos que responden a preocupaciones institucionales y partidarias y resulten tributarios de intereses más políticos que estrictamente periodísticos. 
- Twitter como autopromoción: los periodistas de medios convencionales tweetean sobre comunicación para hacer difusión y promoción de sus propias tareas en los medios y acerca de periodistas o medios afines. Los periodistas digitales hacen lo mismo, pero en menor medida. Esta tendencia aproxima los resultados del trabajo a las reflexiones de Molyneux (2014) y Hanusch y Bruns (2017) sobre la construcción de una imagen de marca a partir de sus tweets y de las interacciones con otros usuarios.

- Brechas en ciertos temas: la escasa presencia del deporte, al contrario de lo que sucede en la totalidad de los medios de masas, responde a una inherente polarización en torno a la política, y al mismo tiempo puede venir dada por el perfil de los periodistas seleccionados en el marco del estudio. La inferencia anterior puede explicar también la brecha entre los contenidos políticos y los de temas sociales, culturales, artísticos, científicos, de interés humano y de sociedad en general. Puede sorprender la escasa atención a los temas económicos, pues se podría suponer que merecerían ser twitteados en cantidades si no iguales por lo menos cercanas a los temas políticos. Si bien hay que considerar esta cuestión con detenimiento, puede aventurarse una razón: la vertiente más polémica, incluso escandalosa, de la actualidad económica en España suele ser difundida bajo parámetros políticos, con lo que viene asimilada temáticamente a la categoría de política. Este aspecto está ligado a la escasa relevancia que los asuntos de la Corona adoptan en ambas muestras y a la desaparición del terrorismo en España como tema de preocupación y asunto de interés general.

- Escasa presencia de lo social: en general, los periodistas digitales se ocupan más de los problemas sociales que los periodistas de medios convencionales. Sin embargo, el estudio concluye que ni unos ni otros aprovechan el enorme potencial de Twitter para difundir asuntos sociales presentes en las capas básicas de la sociedad y que corresponden a preocupaciones tan generalizadas como profundas, según suelen expresar los sondeos de opinión. El escaso margen de diferencia respecto a la mayor atención a temas sociales por parte de los ciberperiodistas que los de medios convencionales podría abrir espacio para inferir una concepción de Twitter e internet abierta a la innovación y al desmarque de los modelos rígidos de la comunicación de masas tal como se dan en España. Pero ese margen se nos asemeja aún insuficiente dados los resultados obtenidos en el análisis del grupo de categorías "Política".

- Terrorismo yihadista: la cuestión del yihadismo aparece como una preocupación notable en el seno de la investigación en su conjunto.

- Más Iberoamérica que Europa: el espectro de países y asuntos internacionales a los que unos y otros periodistas prestan atención es inusitadamente reducido y denota lo que podría considerarse falta de interés ante los intensos procesos de globalización y los cambios civilizatorios que suponen. Se percibe la ausencia de espíritu europeísta y de preocupación por las instituciones europeas, y en cambio, una pronunciada mirada hacia Iberoamérica. Escasea la información y los materiales de debate presentes en la escena internacional, provenientes de importantes centros globales.

- Futuro de los medios y del periodismo: esta reflexión está ausente de los tweets de todos los periodistas del estudio. Existe el tratamiento de cuestiones periodísticas, pero sin un verdadero alcance estratégico.

- La Red habla más de la Red: los periodistas digitales se ocupan más de internet que los periodistas de medios convencionales, pero no reflejan las cuestiones relacionadas con el papel de la Red en la sociedad, los intensos cambios que ella provoca en la sociedad y las perspectivas de futuro inmediato que todo ello supone.

- Cultura como "territorio" olvidado: Todos los periodistas del estudio dedican pocos tweets a asuntos culturales, aunque los ciberperiodistas lo hacen más. En el grupo de categorías "Cultura" se percibe claramente el desaprovechamiento de la oportunidad que Twitter proporciona para tratar una mayor variedad de centros de interés que las que se presentan en los medios convencionales. Dentro del escaso número de tweets

Los periodistas desaprovechan el potencial de Twitter para difundir asuntos sociales presentes en las capas básicas de la sociedad y que se corresponden con las principales preocupaciones de los ciudadanos que reflejan preocupación por los asuntos culturales aún es menor la presencia de interés por asuntos científicos. Tanto periodistas de medios como ciberperiodistas desaprovechan la oportunidad que Twitter proporciona para difundir centros de interés más variados que los que ofrecen los medios convencionales. La categoría "Cultura" supone un espacio en el que se puede percibir con mayor claridad la existencia de incipientes tendencias renovadoras, aperturistas o de futuro.

\section{Bibliografía}

Akter, Tutku; Nweke, Gabriel E. (2016). "Social media users and their social adaptation process in virtual environment: Is it easier for Turkish Cypriots to be social but virtual beings?". Computers in human behavior, v. 61, pp. $472-477$. https://doi.org/10.1016/j.chb.2016.03.067

Alhabash, Saleem; Park, Hyojung; Kononova, Anastasia; Chiang, Yi-Hsuang; Wise, Kevin (2012). "Exploring the motivations of Facebook use in Taiwan". Cyberpsychology, behavior, and social networking, v. 15, n. 6, pp. 304-311. https://doi.org/10.1089/cyber.2011.0611 
Baek, Kanghui; Holton, Avery; Harp, Dustin; Yaschur, Carolyn (2011). "The links that bind: Uncovering novel motivations for linking on Facebook". Computers in human behavior, v. 27, n. 6, pp. 2243-2248.

https://doi.org/10.1016/j.chb.2011.07.003

Bauman, Zygmunt (2003). Modernidad líquida. México: Editorial Fondo de Cultura Económica. ISBN: 9788437507590

Berelson, Bernald (1971). Content analysis in communication research. New York: Hafner. ISBN: 9780028412108

Berganza-Conde, María-Rosa; Ruiz-San-Román, José-Antonio (2005). Investigar en comunicación. Guía práctica de métodos y técnicas de investigación social en comunicación social. Madrid: McGraw Hill. ISBN: 8448198255

Bernal-Bravo, César; Angulo-Rasco, Félix (2013). "Interacciones de los jóvenes andaluces en las redes sociales". Comunicar, v. 40, pp. 25-30.

https://doi.org/10.3916/C40-2013-02-02

Boyd, Danah (2007). "Why youth (heart) social network sites: The role of networked publics in teenage social life". In: Buckingham, David. The John D. and Catherine T. MacArthur Foundation series on digital media and learning. MIT Press, pp. 119-142.

https://www.danah.org/papers/WhyYouthHeart.pdf

Boyd, Danah; Ellison, Nicole B. (2008). "Social network sites: Definition, history and scholarship". Journal of computer-mediated communication, v. 13, n. 1, pp. 210-230.

https://doi.org/10.1111/j.1083-6101.2007.00393.x

Bulut, Zeki-Atil; Doğan, Onur (2017). "The ABCD typology: Profile and motivations of Turkish social network sites users". Computers in human behavior, v. 67, n. 2, pp. 73-83.

https://doi.org/10.1016/j.chb.2016.10.021

Caldevilla-Domínguez, David; Rodríguez-Terceño, José; Barrientos-Báez, Almudena (2019): “El malestar social a través de las nuevas tecnologías: Twitter como herramienta política”. Revista latina de comunicación social, n. 74, pp. 1264-1290. http://www.revistalatinacs.org/074paper/1383/66es.html https://doi.org/10.4185/RLCS-2019-1383-66

Cambronero, Antonio (2012). Twitter. Madrid: Anaya Multimedia. ISBN: 9788441531925

Castells, Manuel (1997). La era de la información. La sociedad red. Madrid: Alianza Editorial. ISBN: 9788420677002

Castells, Manuel (2006). Comunicación y poder. Madrid: Alianza Editorial. ISBN: 9788420684994

Cheung, Christy M. K.; Chiu, Pui-Yee; Lee, Matthew K. O. (2011). “Online social networks: Why do students use Facebook?". Computers in human behavior, v. 27, n. 4, pp. 1337-1343. https://doi.org/10.1016/j.chb.2010.07.028

Chung, Te-Li; Anaza, Nwamaka A.; Park, Joohyung; Hall-Phillips, Adrienne (2016). "Who's behind the screen? Segmenting social venture consumers through social media usage". Journal of retailing and consumer services, v. 28, pp. 288-295. https://doi.org/10.1016/j.jretconser.2015.01.006

Colás-Bravo, Pilar; González-Ramírez, Teresa; De-Pablos-Pons, Juan (2013). "Juventud y redes sociales: motivaciones y usos preferentes". Comunicar, v. 40, pp. 15-23.

https://doi.org/10.3916/C40-2013-02-01

Del-Fresno-García, Miguel; Marqués-Martínez, Pilar; Sánchez-Paunero, David (eds.) (2014). Conectados por redes sociales. Introducción al análisis de redes y casos prácticos. Barcelona: Editorial UOC. ISBN: 9788490642344

Gentile, Brittany; Twenge, Jean M.; Freeman, Elise C.; Campbell, W. Keith (2012). "The effect of social networking websites on positive self-views: An experimental investigation". Computers in human behavior, v. 28, n. 5, pp. 1929-1933. https://doi.org/10.1016/j.chb.2012.05.012

Galindo-Cáceres, Jesús (2010a). “La internet y sus redes sociales. Comunicología e ingeniería en Comunicación Social de un fenómeno aún emergente". Razón y palabra, 71. http://www.razonypalabra.org.mx/N/N71/VARIA/34\%20GALINDO_REVISADO.pdf

Galindo-Cáceres, Jesús (2010b). "El nuevo nicho civilizatorio del ciberespacio: La red social como un desarrollo de la cibercultura". Revista mexicana de comunicación, v. 23, n. 123, pp. 24-26.

https://dialnet.unirioja.es/servlet/articulo?codigo $=5670263$

Galindo-Cáceres, Jesús (2011). “Comunicación, vida y sociedad. La sociogenética de la Teoría de la Comunicación frente a la paleontologenética de la Comunicación Humana”. Chasqui: Revista latinoamericana de comunicación, n. 114-115, pp. 66-68.

https://revistachasqui.org/index.php/chasqui/article/view/560

https://doi.org/10.16921/chasqui.v0i114-115.560 
Gillmor, Dan (2004). We, the media. Sebastopol, CA: O'Reilly Media. ISBN: 9780596102272

Granovetter, Mark (1974). Getting a job. A study on contacts and careers. Cambridge, MA: Harvard University. ISBN: 978 0226305813

Gutiérrez-Rubí, Antoni (2013). Otro partido es posible. Madrid: Fundación Ideas. ISBN: 9788493906689

Hanusch, Folker; Bruns, Axel (2017). “Journalistic branding on Twitter. A representative study of Australian journalists' profile descriptions". Digital journalism, v. 5, n. 1, pp. 26-43.

https://doi.org/10.1080/21670811.2016.1152161

Holsti, Ole R. (1969). Content analysis for the Social Sciences and Humanities. Reading, MA: Addison-Wesley. ISBN: 978 0201029406

Jaraba, Gabriel (2013). Usos periodísticos de Twitter. Cómo emplean los periodistas catalanes las posibilidades informativas del microblogging. Campus de Bellaterra: Universidad Autónoma de Barcelona, Departamento de Periodismo y Ciencias de la Comunicación.

Jaraba, Gabriel (2014). Periodismo en internet. Cómo escribir y publicar contenidos de calidad en la Red. Barcelona: Robinbook. ISBN: 9788415256694

Jaraba, Gabriel (2015). Twitter para periodistas. Barcelona: Editorial UOC. ISBN: 9788490647264

King, Brett; Wertheimer, Michael (2009). Max Wertheimer \& Gestalt theory. New Brunswick and London: Transaction. ISBN: 9781412807180

Krippendorff, Klaus (1990). Metodología de análisis de contenido. Teoría y práctica. Barcelona: Paidós. ISBN: 97884 75096278

Kurtulus, Sema; Ozkan, Erdem; Öztürk, Selen (2015). "How do social media users in Turkey differ in terms of their use habits and preferences?". International journal of business and information, v. 10, pp. 337-364.

https://ijbi.org/ijbi/article/view/118

Lenhart, Amanda; Madden, May; Macgill, Alexandra-Rankin; Smith, Aaron (2007). Teens and social media. The use of social media gains a greater foothold in teen life as they embrace the conversational nature of interactive online media. Pew Internet \& American Life Project.

http://pewinternet.org/PPF/r/230/report_display.asp

Lévy, Pierre (1999). ¿Qué es lo virtual? Barcelona: Paidós Ibérica. ISBN: 9788449305856

Lévy, Pierre (2007). Cibercultura: la cultura de la sociedad digital. Barcelona: Anthropos. ISBN: 9788476588086

Lewin, Kurt (1947). "Frontiers in group dynamics: II. Channels of group life; Social planning and action research". Human relations, v. 1, p. 143-153.

http://www.sagepub.com/mertler2study/articles/Lewin.pdf

Lin, Kuan-Yu; Lu, Hsi-Peng (2011). "Why people use social networking sites: An empirical study integrating network externalities and motivation theory". Computers in human behavior, v. 27, n. 3, pp. 1152-1161.

https://doi.org/10.1016/j.chb.2010.12.009

López-Meri, Amparo (2015). "El impacto de Twitter en el periodismo: un estado de la cuestión". Revista de la Asociación Española de Investigación de la Comunicación, v. 2, n. 4, pp. 34-41.

http://www.revistaeic.eu/index.php/raeic/article/view/55

Manovich, Lev (2005). El lenguaje de los nuevos medios de comunicación: la imagen en la era digital. Barcelona: Paidós. ISBN: 9788449317699

Martín-Barbero, Jesús (2015). “¿Desde dónde pensamos la comunicación hoy?” Chasqui: Revista latinoamericana de comunicación, v. 128, pp. 13-29.

https://revistachasqui.org/index.php/chasqui/article/view/2545

Martín-Serrano, Manuel (2008). La mediación social. Madrid: Akal. ISBN: 9788446028963

Martínez-Serrano, María-del-Carmen; Ferraz-da-Cunha, Elisabete (2016). “Uso de las redes sociales por los alumnos universitarios de educación: un estudio de caso de la Península Ibérica”. Tendencias pedagógicas, v. 28, pp. 33-44. https://doi.org/10.15366/tp2016.28.003

Matsa, Katherina-Eva; Mitchell, Amy (2014). "8 keys takeaways about social media and news". Pew Research Center. Journalim \& media, 26 March.

http://www.journalism.org/2014/03/26/8-key-takeaways-about-social-media-and-news 
McCombs, Maxwell (1992). "Explorers and surveyors: Expanding strategies for agenda-setting research". Journalism quarterly, v. 69, n. 4, pp. 813-824.

https://doi.org/10.1177/107769909206900402

McCombs, Maxwell (1994). "The future agenda for agenda-setting research". Journal of mass communication studies, n. 45, pp. 171-181. https://doi.org/10.24460/mscom.45.0_171

McCombs, Maxwell; Evatt, Dixie (1995). “Los temas y los aspectos: explorando una nueva dimensión de la agenda-setting". Comunicación y sociedad, v. 8, n. 1, pp. 7-32.

https://revistas.unav.edu/index.php/communication-and-society/article/view/35616 https://doi.org/10.15581/003.8.1.7-32

McCombs, Maxwell; Masel-Walters, Lynne (1976). "Agenda-setting: A new perspective on mass communication". Mass communication review, v. 2, n. 3, pp. 3-7.

McCombs, Maxwell; Shaw, Donald L. (1972). "The agenda-setting function of the mass media”. Public opinion quarterly, n. 36, pp. 176-187.

McCombs, Maxwell; Shaw, Donald L. (1976). "Structuring the unseen unvironment". Journal of communication, n. 26, n. 2, pp. 18-22.

https://doi.org/10.1111/j.1460-2466.1976.tb01374.x

Molyneux, Logan (2014). "What journalists retweet: Opinion, humor, and brand development on Twitter". Journalism, v. 16, n. 1, pp. 1-16.

https://doi.org/10.1177/1464884914550135

Moreno, Jacob L. (1954). Who shall survive? Foundations of sociometry, group psychotherapy and sociodrama. Boston: Beacon House. ISBN: 9789992695722

Nafría, Ismael (2007). Web 2.0. El usuario, el nuevo rey de internet. Barcelona: Gestión 2000. ISBN: 9788498750034

O'Keeffe, Gwenn-Schurgin; Clarke-Pearson, Kathleen (2011). "The impact of social media on children, adolescents, and families". Pediatrics, v. 127, n. 4, pp. 800-804.

https://doi.org/10.1542/peds.2011-0054

Orihuela, José-Luis (2011). Mundo Twitter. Barcelona: Alienta. ISBN: 9788492414895

Orihuela, José-Luis (2015). Los medios después de internet. Barcelona: Editorial UOC. ISBN: 9788490647004

Papacharissi, Zizi; Mendelson, Andrew (2011). "Toward a new(er) sociability: Uses, gratifications and social capital on Facebook". En: Papathanassopoulos, Stylianos (ed.). Media perspectives for the $21^{\text {st }}$ century. Routledge: New York, pp. 212-230. ISBN: 9780203834077

Pempek, Tiffany A.; Yermolayeva, Yevdokiya A.; Calvert, Sandra L. (2009) “College students' social networking experiences on Facebook". Journal of applied developmental psychology, v. 30, n. 3, pp. 227-238.

Pérez-Tornero, José-Manuel; Tejedor, Santiago; Simelio-Solà, Núria; Marín-Ochoa, Beatríz-Elena (2015). "Estudiantes universitarios ante los retos formativos de las redes sociales: el caso de Colombia". Estudios sobre el mensaje periodístico, v. 21, n. 1, pp. 509-521.

https://doi.org/10.5209/rev_ESMP.2015.v21.n1.49108

Pisani, Francis; Piotet, Dominique (2009). La alquimia de las multitudes. Barcelona: Paidós. ISBN: 9788449321962

Piscitelli, Alejandro (2005). Internet, la imprenta del siglo XXI. Barcelona: Gedisa. ISBN: 9788497840606

Piscitelli, Alejandro; Adaime, Iván; Binder, Inés (2010). El proyecto Facebook y la posuniversidad. Sistemas operativos sociales y entornos abiertos de aprendizaje. Barcelona: Ariel.

http://www.codajic.org/sites/www.codajic.org/files/El\%20Proyecto\%20Facebook.pdf

Quan-Haase, Anabel; Young, Alison (2010). “Uses and gratifications of social media: A comparison of Facebook and instant messaging". Bulletin of science, technology \& society, v. 30, n. 5, pp. 350-361.

https://doi.org/10.1177/0270467610380009

Reig, Ramón (2010). La telaraña mediática. Sevilla: Comunicación Social Ediciones. ISBN: 9788492860548

Reig, Ramón (2011). Los dueños del periodismo. Barcelona: Gedisa. ISBN: 9788497846189

Reig, Ramón; Mancinas-Chávez, Rosalba; Nogales-Bocio, Antonia (2017). “Enfoque estructural complejo: propuesta metodológica desde el periodismo". Estudios sobre el mensaje periodístico, v. 23, n. 1, pp. 191-208.

https://doi.org/10.5209/ESMP.55591 
Rheingold, Howard (2002). Multitudes inteligentes. Barcelona: Gedisa. ISBN: 9788497840620

Ruano, Luis E.; Congote, Ernesto L.; Torres, Andrés E. (2016). “Comunicación e interacción por el uso de dispositivos tecnológicos y redes sociales virtuales en estudiantes universitarios". Revista ibérica de sistemas e tecnologias de informação, n. 19, pp. 15-31.

https://dialnet.unirioja.es/servlet/articulo?codigo $=6669606$

Sandiumenge, Lali (2012). Guerrillers del teclat. Barcelona: La Magrana. ISBN: 9788482642765

Schwarz, Ori (2011). "Who moved my conversation? Instant messaging, intertextuality and new regimes of intimacy and truth". Media culture society, v. 33, n. 1, pp. 71-87.

https://doi.org/10.1177/0163443710385501

Shao, Guosong (2009). "Understanding the appeal of user-generated media: A uses and gratification perspective". Internet research, v. 19, n. 1, pp. 7-25.

https://doi.org/10.1108/10662240910927795

Sharma, Rahul; Verma, Rajesh (2015). "Social networkers' motivation to use online social networking sites: Implications for leveraging digital marketing". International journal of applied business and economic research, v. 13, n. 3, pp. 11591171.

https://serialsjournals.com/abstract/82876_11.pdf

Sheldon, Pavica (2009). "Maintain or develop new relationships? Gender differences in Facebook use". Rocky mountain communication review, v. 6, n. 1, pp. 51-56.

Singh, Jagtar; Grizzle, Alton; Joan-Yee, Sin; Hope-Culver, Sherry (eds.) (2015). Media and information literacy for the sustainable development goals. MILID Yearbook. Gothenburg: Nordicom; University of Gothenburg.

http://www.nordicom.gu.se/sites/default/files/publikationer-hela-pdf/milid_yearbook_2015.pdf

Tejedor, Santiago; Carniel-Bugs, Ricardo; Giraldo-Luque, Santiago (2018). "Los estudiantes de Comunicación en las redes sociales: estudio comparativo entre Brasil, Colombia y España”. Transinformação, v. 30, n. 2, pp. $267-276$.

https://doi.org/10.1590/2318-08892018000200010

Thompson, John B. (1998). Los media y la modernidad. Barcelona: Paidós. ISBN: 9788449305788

Vilches, Lorenzo (coord.) (2011). La investigación en comunicación. Métodos y técnicas en la era digital. Barcelona: Gedisa. ISBN: 9788497846691

\section{Colección EPI Scholar}

\section{Libros científicos de Información, Documentación y Comunicación}

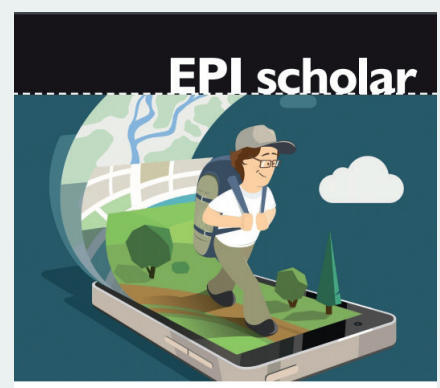

TURISMO RURAL ONLINE PÁGINAS WEB Y REDES SOCIALES GERSÓN BELTRÁN

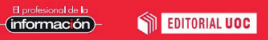

\section{Turismo rural online. Páginas web y redes sociales de Gersón Beltrán}

Los turistas están conectados en todo momento y se mueven en un entorno social, local y móvil: buscan información en internet antes de ir a su destino y realizan las reservas por anticipado, utilizan el móvil durante su experiencia turística para comunicarse y, tanto durante como tras el viaje, comparten sus opiniones en medios sociales.

Las empresas y destinos turísticos deben adaptarse a esta realidad, en la que lo físico es inseparable de lo online, conformando un entorno híbrido. Paralelamente, los espacios rurales sufren numerosos problemas de desarrollo y despoblación, y el turismo rural constituye una actividad que puede ayudar a revertir esta situación. Las nuevas tecnologías se configuran como herramientas que unen a los turistas conectados con estos espacios.

Este libro ofrece una visión general de la situación del turismo rural online, así como las claves para que las empresas y destinos turísticos logren conectar con los turistas y éstos disfruten de las experiencias únicas que les ofrece el turismo rural.

Beltrán, Gersón (2019). Turismo rural online. Páginas web y redes sociales. Barcelona: El profesional de la información, Editorial UOC, colección EPI Scholar n. 11, 170 pp. ISBN: 9788491803881

Información

http://www.elprofesionaldelainformacion.com/librosEPIScholar.html 


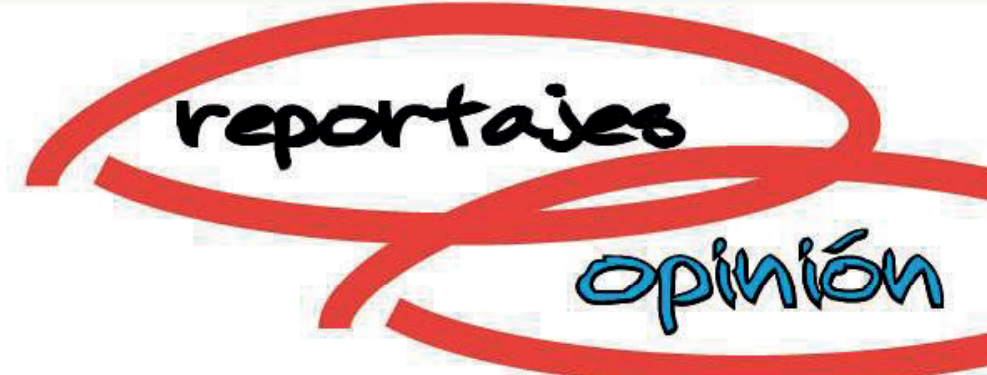

\section{biblidbuses}

\section{Redas soclalas}

actualidad

\section{publicas}

\section{entrevistas}

\section{universitarias}

Suscríbete 